Candidate single nucleotide polymorphisms and thromboembolism in acute lymphoblastic leukemia - A NOPHO ALL2008 study

\author{
Jarvis, Kirsten Brunsvig
}

2019-12

Jarvis , K B , LeBlanc , M , Tulstrup , M , Nielsen , R L , Albertsen , B K , Gupta , R , Huttunen, P , Jonsson, O G , Rank , C U , Ranta , S, Ruud, E, Saks , K, Trakymiene , S S , Tuckuviene, R \& Schmiegelow, K 2019 , ' Candidate single nucleotide polymorphisms and thromboembolism in acute lymphoblastic leukemia - A NOPHO ALL2008 study ' , Thrombosis Research , vol. 184 , pp. 92-98 . https://doi.org/10.1016/j.thromres.2019.11.002

http://hdl.handle.net/10138/323892

https://doi.org/10.1016/j.thromres.2019.11.002

publishedVersion

Downloaded from Helda, University of Helsinki institutional repository.

This is an electronic reprint of the original article.

This reprint may differ from the original in pagination and typographic detail.

Please cite the original version. 
Full Length Article

\title{
Candidate single nucleotide polymorphisms and thromboembolism in acute lymphoblastic leukemia - A NOPHO ALL2008 study
}

\author{
Kirsten Brunsvig Jarvis ${ }^{\mathrm{a}, \mathrm{b}, \mathrm{c}, *}$, Marissa LeBlanc ${ }^{\mathrm{d}}$, Morten Tulstrup ${ }^{\mathrm{e}}$, Rikke Linnemann Nielsen ${ }^{\mathrm{f}, \mathrm{g}}$, \\ Birgitte Klug Albertsen $^{\mathrm{h}}$, Ramneek Gupta ${ }^{\mathrm{f}}$, Pasi Huttunen ${ }^{\mathrm{i}}$, Ólafur Gisli Jónsson ${ }^{\mathrm{j}}$, \\ Cecilie Utke Rank ${ }^{\mathrm{k}, \mathrm{l}}$, Susanna Ranta ${ }^{\mathrm{m}, \mathrm{n}}$, Ellen Ruud ${ }^{\mathrm{a}, \mathrm{b}, \mathrm{c}}$, Kadri Saks ${ }^{\mathrm{o}}$, Sonata Saulyte Trakymiene ${ }^{\mathrm{p}}$, \\ Ruta Tuckuviene $^{\mathrm{q}}$, Kjeld Schmiegelow ${ }^{\mathrm{e}, \mathrm{r}}$
}

${ }^{a}$ Department of Pediatric Hematology and Oncology, Oslo University Hospital, Postboks 4950, Nydalen, 0424 Oslo, Norway

${ }^{\mathrm{b}}$ Department of Pediatric Research, Oslo University Hospital, Postbok 4950, Nydalen, 0424 Oslo, Norway

${ }^{\mathrm{c}}$ The Faculty of Medicine, Institute of Clinical Medicine, University of Oslo, Postboks 1072, Blindern, 0316 Oslo, Norway

${ }^{\mathrm{d}}$ Oslo Center for Biostatistics and Epidemiology, Oslo University Hospital, Postboks 4950, Nydalen, 0424 Oslo, Norway

${ }^{\mathrm{e}}$ Department of Pediatrics and Adolescent Medicine, Rigshospitalet, University Hospital of Copenhagen, Belgdamsvej 9, 2100 Copenhagen, Denmark

${ }^{\mathrm{f}}$ Department of Health technology, Technical University of Denmark, 2800 Kgs Lyngby, Denmark

${ }^{\mathrm{g}}$ Sino-Danish Center for Education and Research, University of Chinese Academy of Sciences, 380 Huaibeizhuang, Huairou district, Beijing, China

${ }^{\mathrm{h}}$ Children and Adolescent Health, Aarhus University Hospital, Palle Juul-Jensens Boulevard 99, 8200 Aarhus, Denmark

${ }^{i}$ Department of Pediatric Hematology, Oncology and Stem Cell Transplantation, New Children's Hospital, Helsinki University Hospital, Stenbäckinkatu 9, o0290 Helsinki, Finland

${ }^{\mathrm{j}}$ Children's Hospital, Barnaspitali Hringsins, Landspitali University Hospital, Hringbraut 101, 101 Reykjavik, Iceland

${ }^{\mathrm{k}}$ Department of hematology, Rigshospitalet, University Hospital of Copenhagen, Belgdamsvej 9, 2100 Copenhagen, Denmark

${ }^{1}$ Pediatric Oncology Research Laboratory, Rigshospitalet, University of Copenhagen, Belgdamsvej 9, 2100 Copenhagen, Denmark

${ }^{\mathrm{m}}$ Department of Women's and Children's Health, Karolinska University Hospital, Eugeniavägen 3, 17176 Solna, Sweden

${ }^{n}$ Childhood Cancer Research Unit, Women's and Children's Health, Karolinska Insitutet, Solnavägen 1, 17177 Solna, Sweden

${ }^{\circ}$ Department of Hematology and Oncology, Tallinn Children's Hospital, 13419 Tallinn, Estonia

${ }^{\mathrm{P}}$ Center for Pediatric Oncology and Hematology, Children's Hospital, Vilnius University Hospital Santaros Klinikos and Vilnius University, Vilnius 08410, Lithuania

${ }^{\mathrm{q}}$ Department of Pediatrics, Aalborg University Hospital, Hobrovej 18-22, 9100 Aalborg, Denmark

${ }^{\mathrm{r}}$ Institute of Clinical Medicine, Faculty of Medicine, University of Copenhagen, Nørregade 10, 1165 Copenhagen, Denmark

A R T I C L E IN F O

\section{Keywords:}

Thromboembolism

Single nucleotide polymorphisms

Acute lymphoblastic leukemia

\begin{abstract}
A B S T R A C T
Introduction: Thromboembolism is a serious toxicity of acute lymphoblastic leukemia treatment, and contributes to substantial morbidity and mortality. Several single nucleotide polymorphisms have been associated with thromboembolism in the general population; however, their impact in patients with acute lymphoblastic leukemia, particularly in children, remains uncertain.

Materials and methods: We collected constitutional DNA and prospectively registered thromboembolic events in 1252 patients, 1-45 years, with acute lymphoblastic leukemia included in the Nordic Society of Pediatric Hematology and Oncology ALL2008 protocol in the Nordic and Baltic countries (7/2008-7/2016). Based on previously published data and a priori power calculations, we selected four single nucleotide polymorphisms: $F 5$ rs6025, F11 rs2036914, FGG rs2066865, and $A B O$ rs8176719.

Results: The 2.5 year cumulative incidence of thromboembolism was $7.1 \%$ ( $95 \%$ confidence interval (CI) 5.6-8.5). F11 rs2036914 was associated with thromboembolism (hazard ratio (HR) 1.52, 95\%CI 1.11-2.07) and there was a borderline significant association for $F G G$ rs2066865 (HR 1.37, 95\%CI 0.99-1.91), but no association for $A B O$ rs 8176719 or $F 5$ rs6025 in multiple cox regression. A genetic risk score based on $F 11$ rs2036914 and $F G G$ rs2066865 was associated with thromboembolism (HR 1.45 per risk allele, 95\%CI 1.15-1.81), the association was strongest in adolescents $10.0-17.9$ years (HR 1.64).
\end{abstract}

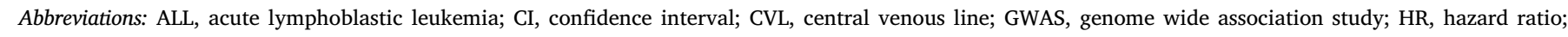

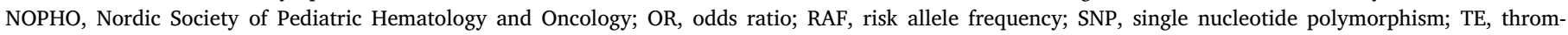
boembolism

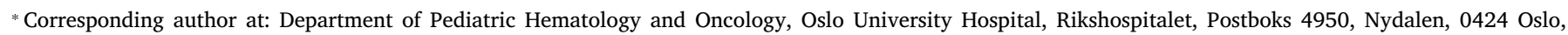
Norway.

E-mail address: kirjar@ous-hf.no (K.B. Jarvis). 
Conclusion: If validated, a $F 11$ rs2036914/FGG rs2066865 risk prediction model should be tested as a stratification tool for prevention of thromboembolism in patients with acute lymphoblastic leukemia.

\section{Introduction}

Children, adolescents, and adults with acute lymphoblastic leukemia (ALL) are at increased risk of developing thromboembolism (TE), a common serious toxicity of ALL treatment. A recent study from Nordic Society of Pediatric Hematology and Oncology (NOPHO) found a 7.9\% cumulative incidence of TE in patients with ALL, with age being the most important risk factor [1]. The NOPHO ALL2008 protocol has uniform ALL treatment for both children and adults, 1-45 years old. The protocol includes mandatory prospective toxicity registration, including TE, with high registration compliance [2].

Several genome wide-association studies (GWAS) have been performed on TE in adults. In 2015, a meta-analysis of GWAS on TE found six well-known genome-wide significant loci that contributed to increased risk of TE ( $A B O, F 2, F 5, F 11, F G G$, and PROCR), and three new loci of which two were replicated in a validation study (TSPAN15 and SLC44A2) [3]. In 2012, de Haan et al. [4] tested 31 single nucleotide polymorphisms (SNP) known to be associated with TE and found that the top five SNPs F5 rs6025, F2 rs1799963, ABO rs8176719, FGG rs2066865, and F11 rs2036914 predicted TE almost as well as a genetic risk score based on all 31 SNPs. Folsom et al. [5] recently replicated the five SNP genetic risk score by de Haan in white Americans, with a 1.41fold increased risk of venous TE per allele increment.

F5 rs6025 is known as the factor V Leiden mutation, which causes activated protein $C$ resistance, and F2 rs1799963 is known as the prothrombin mutation, which causes increased prothrombin (factor II) levels [6]. The major allele of $A B O$ rs8176719 is a deletion that predicts blood group $O$ [7], and the risk allele has been associated with both venous TE [8] and with cerebral sinus vein thrombosis [9] in noncancer adult studies. Genetic variation in the $F G G$ gene, such as rs2066865, has been associated with different levels of isoforms of the fibrinogen gamma chain and with TE development [10]. Several SNPs in the F11 gene, including rs2036914, have been associated with increased levels of factor XI and TE development [11]. Patients with ALL are already at increased risk of TE due to the leukemia itself, the chemotherapy (e.g. asparaginase and steroids), the presence of central venous lines (CVL), and other well-defined non-genetic prothrombotic risk factors, including older age. However, little data exists on the impact of the above-mentioned SNPs on TE development in patients with ALL, particularly in children.

Developing a tool to identify patients with ALL at risk of TE who would benefit from thromboprophylaxis may have the potential to reduce both morbidity and mortality. In this respect, we aimed to identify the role of well-defined SNPs on the development of TE in children and adults with ALL. We also studied multi-SNP genetic risk scores for the detection of TE. We hypothesized that F5 rs6025, ABO rs8176719, FGG rs2066865, and $F 11$ rs2036914 contributed to the risk of TE development in the large cohort of children and adults with ALL treated according to the NOPHO ALL2008 protocol.

\section{Materials and methods}

From 7/2008 to 7/2016 patients with ALL included in the NOPHO ALL2008 protocol in Denmark, Estonia, Finland, Iceland, Lithuania, Norway, and Sweden were invited to participate in genetic add-on studies. The NOPHO ALL2008 protocol was approved by the National Medicines Agencies and the relevant national or regional ethical committees in each participating country. The genetic add-on study required additional informed consent and was approved by the ethical committees in the participating countries. The study was conducted in accordance with the Declaration of Helsinki.

The NOPHO ALL2008 protocol has been described in detail elsewhere [1,2,12-14]. Data on patient demographics were collected from the NOPHO ALL registry on October 10th 2017. DNA was sampled from 1812 patients, of which 560 were excluded on genetic or clinical criteria (Fig. 1), resulting in 1252 patients in this genetic study.

The NOPHO ALL2008 protocol has mandatory prospective toxicity registration, including TE [2]. TE was defined as first symptomatic arterial or venous TE verified by imaging or asymptomatic arterial or venous $\mathrm{TE}$ requiring anticoagulation treatment-diagnosed by imaging due to other non-TE related symptoms. The date of TE was defined as the date of diagnostic imaging or the date of death if diagnosed at autopsy.

SNP profiling of post-remission DNA was done using the Omni

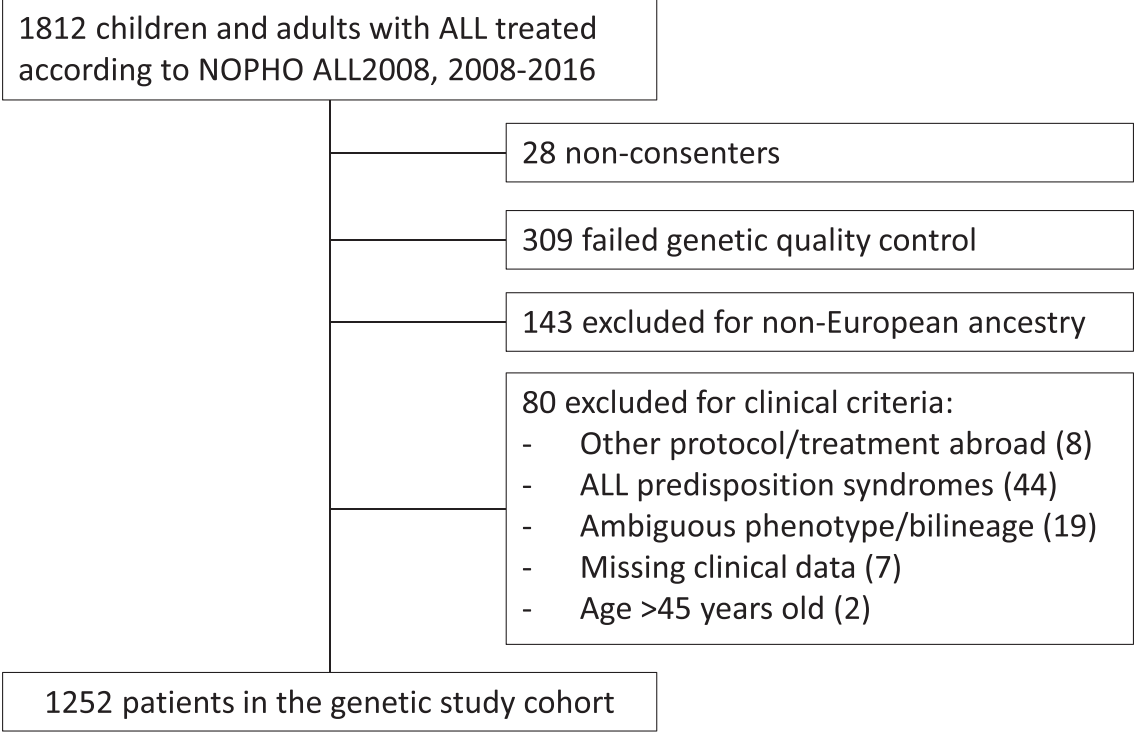

Fig. 1. Flowsheet. 
2.5exome-8-BeadChip arrays (Illumina, San Diego, CA, USA). Standard quality control procedures were performed according to previously published criteria (supplementary material) [15]. F2 rs1799963 and $A B O$ rs8176719 were imputed, but the F2 rs1799963 imputation did not reach the quality threshold of 0.7 (Table 1 ) and was excluded.

We performed a priori power calculations for cox regression of SNPassociation testing (Table 1) [16]. In cox regression analysis of time to TE event, patients were censored at end of ALL treatment (120 weeks), date of hematopoietic stem cell transplantation, loss to follow-up, date of data collection (October 10th 2017), or date of a competing event (death, relapse, or second malignant neoplasm), whichever came first. The continuous variable age was categorized to three groups: 1.0-9.9, $10.0-17.9$, and $18.0-45.9$ years. The clinical covariates age, sex, presence of mediastinal mass, and enlarged lymph nodes at diagnosis were kept as covariates in the genetic analyses, unless otherwise noted, and we included the first two genetic principal components from the full Illumina SNP data to control for population stratification. The SNPs were analyzed using an additive genetic model. A sensitivity analysis was performed by restricting the analysis to the period of asparaginase exposure (supplementary material). Separate cox regression models were set up for the competing events. Cumulative incidence estimates were compared with Gray's test.

In exploratory analysis, we created unweighted, additive genetic risk scores defined as the number of risk alleles in each individual. We explored genetic risk scores using all four SNPs and using only the nominally significant SNPs in our study. A cox model with an interaction term between age and the genetic risk score was investigated.

For all cox regression analyses the assumption of proportional hazards was examined using Schoenfelt residuals. Hazard rations (HR) are presented with $95 \%$ confidence intervals (CI) and two-sided $p$-values $<.05$ are considered statistically significant. All statistical analyses were performed using $\mathrm{R}$ computing software, version 3.4.3.

\section{Results}

\subsection{Clinical characteristics}

During ALL treatment 89 of 1252 patients developed major TE (2.5 year cumulative incidence $7.1 \%, 95 \%$ CI 5.6-8.5) at a median of 89 days (50\% range: $52-127$ days) from diagnosis. The 2.5 -year cumulative incidence for children 1.0-9.9 years was 3.8\% (95\% CI 2.6-5.1), for adolescents 10.0-17.9 years $14.4 \%$ (95\% CI 9.9-19.0), and for adults $18.0-45.9$ years $16.7 \%$ (95\% CI 10.2-23.3). Fifty TE events (56.2\% of all cases) were deep vein thromboses, of which one was a combined arterial and venous event in the portal hepatic system, 25 (28.1\%) were cerebral sinus vein thromboses, and 14 (15.7\%) were pulmonary embolisms. Forty-one TE events (46.1\%, 95\% CI 35.6-56.9) were reported to be CVL-related. Seventy-six TE events (85.4\%, 95\% CI 76.0-91.7) occurred during asparaginase exposure, while six $(6.7 \%$, 95\% CI 2.8-14.6) occurred during induction therapy. Baseline clinical characteristics of patients with and without TE development are presented in Table 2. In multiple cox regression analysis of time to TE event, we found significant associations for age and enlarged lymph nodes, with a trend to association for mediastinal mass. The patient demographics and the significant associations were comparable to those previously reported from the NOPHO group by Rank et al. [1].

\subsection{Genetic variables}

For each of the predefined TE-associated SNPs, we had power ranging from $48 \%$ to $96 \%$, assuming the SNP-specific effect sizes and risk allele frequencies found in the review by Morange et al. [17] from 2015. The risk allele frequencies of the selected SNPS in the study population are provided in Table 1. Results of the single SNP and multiple cox regression analysis are displayed in Table 3. We found a significant association with TE development for $F 11$ rs2036914 (HR 1.52, 95\% CI 1.11-2.07) and a trend to association for FGG rs2066865 (HR 1.37, 95\% CI 0.99-1.91). We found no association for $F 5$ rs6025 or $A B O$ rs8176719 with the point estimates of the HR close to 1 . None of the three patients with two risk alleles of $F 5$ rs6025 (homozygous factor V Leiden mutation) developed TE. Patients with one risk allele of F5 rs6025 had a cumulative incidence of TE of 6.7\% (95\% CI 3.8-9.7), compared to a cumulative incidence of $7.1 \%$ (95\% CI 5.6-8.6) among patients with no risk alleles (Table 4).

HR results did not change markedly when only analyzing patients during asparaginase exposure, with a significant association for F11 rs2036914 (HR 1.66, 95\% CI 1.18-2.35) and a trend to association for FGG rs2066865 (HR 1.36, 95\% CI 0.95-1.96), but no association for F5 rs6025 or $A B O$ rs8176719 in multiple cox regression (Supplementary Table S1).

In the single-SNP analyses we set up one cox model per SNP, while in the multiple-SNP analysis all four SNPs were included in one cox model. In all the cox regression analyses we controlled for age, sex, mediastinal mass, enlarged lymph nodes, and the first two principal components of the genetic data.

In our study population, competing risks were scarce; the majority occurring after the TE events (Supplementary Fig. S1). We found no associations between our individual SNPs and the competing risks; regarding F5 rs6025, the numbers became too small for statistical testing. There were no significant associations between our individual SNPs and the compound competing risk (Supplementary Table S2).

\subsection{Genetic risk score}

In exploratory analysis, an unweighted additive 2-SNP genetic risk score based on $F 11$ rs2036914 and FGG rs2066865 was significantly associated with TE (HR 1.45 per risk allele, 95\% CI 1.15-1.81, p 0.001). Fig. 2 shows an increasing incidence of TE with increasing number of risk alleles. Twenty-two of 119 patients with $\geq 3$ risk alleles of the 2SNP genetic risk score developed TE (cumulative incidence 11.1\%, 95\%

Table 1

Chosen SNPs.

\begin{tabular}{|c|c|c|c|c|c|c|c|c|}
\hline Name & $\begin{array}{l}\text { Expected } \mathrm{RAF}^{\mathrm{a}} \text { from } \\
\text { literature }\end{array}$ & $\begin{array}{l}\text { Estimated } \mathrm{OR}^{\mathrm{a}} \text { from } \\
\text { literature }\end{array}$ & Power $^{\mathrm{b}}$ & $\begin{array}{l}\text { Imputation info } \\
\text { score }^{c}\end{array}$ & Nucleotides & $\begin{array}{l}\text { Genotype } \\
\text { distribution }^{\mathrm{d}}\end{array}$ & Observed RAF & $\begin{array}{l}\text { Missing genotype } \\
\text { count }\end{array}$ \\
\hline F5 rs6025 & 0.05 & 3.00 & 0.96 & - & $\mathrm{CC} / \mathrm{CT} / \mathrm{TT}$ & $1174 / 75 / 3$ & 0.03 & 0 \\
\hline$A B O$ rs8176719 & 0.39 & 1.50 & 0.74 & 0.999 & $-/-\mathrm{C} / \mathrm{CC}$ & $450 / 586 / 212$ & 0.40 & 4 \\
\hline$F G G$ rs2066865 & 0.25 & 1.47 & 0.61 & - & GG/GA/AA & $685 / 478 / 89$ & 0.26 & 0 \\
\hline F11 rs2036914 & 0.52 & 1.35 & 0.48 & - & TT/TC/CC & $268 / 619 / 365$ & 0.54 & 0 \\
\hline F2 rs1799963 & 0.01 & 2.50 & 0.48 & 0.579 & GG/GA/AA & $1232 / 3 / 0$ & 0.001 & 17 \\
\hline
\end{tabular}

${ }^{\text {a }}$ Risk allele frequency (RAF) and estimated odds ratio (OR) from the 2015 review by Morange et al. [17]. The RAF for rs8176719 was taken from the European population in the 1000 genomes project, Ensembl release 96 [18].

${ }^{\mathrm{b}}$ Power calculation for cox regression of SNP-association testing, estimated total N 1200 and incidence $7 \%$.

c Not relevant for directly genotyped SNPs.

d The genotype distribution is given as $0 / 1 / 2$ risk alleles. 
Table 2

Clinical characteristics of patients with and without TE development.

\begin{tabular}{|c|c|c|c|c|c|c|}
\hline Patient characteristics & & $\begin{array}{l}\text { Without TE } \\
\text { N (\%) }\end{array}$ & $\begin{array}{l}\text { With TE } \\
\text { N (\%) }\end{array}$ & HR & $95 \% \mathrm{CI}$ & $\mathrm{p}$-Value ${ }^{\mathrm{a}}$ \\
\hline \multirow[t]{3}{*}{ Age } & $1.0-9.9$ years & 860 (73.9) & $34(38.2)$ & Ref. & & \\
\hline & $1.0-17.9$ years & $197(17.0)$ & $34(38.2)$ & 3.84 & $2.36-6.24$ & $<0.0001$ \\
\hline & 18.0-45.9 years & $106(9.1)$ & $21(23.6)$ & 4.12 & $2.30-7.40$ & $<0.0001$ \\
\hline \multirow[t]{2}{*}{ Sex } & Male & $644(55.4)$ & $54(60.7)$ & Ref. & & \\
\hline & Female & $519(44.6)$ & $35(39.3)$ & 0.98 & $0.63-1.54$ & 0.97 \\
\hline \multirow[t]{2}{*}{ Phenotype } & Precursor-B cell & $1008(86.7)$ & $65(73.0)$ & Ref. & & \\
\hline & T-cell & $155(13.3)$ & $24(27.0)$ & 1.19 & $0.36-3.90$ & 0.77 \\
\hline \multirow[t]{3}{*}{ Induction } & Prednisone & $931(80.1)$ & $61(68.6)$ & Ref. & & \\
\hline & Dexamethasone & $221(19.0)$ & $27(30.3)$ & 0.69 & $0.25-1.87$ & 0.46 \\
\hline & Missing & $11(0.9)$ & $1(1.1)$ & - & & \\
\hline \multirow[t]{3}{*}{ Mediastinal mass } & No & $1073(92.3)$ & $70(78.6)$ & Ref. & & \\
\hline & Yes & $79(7.8)$ & $16(18.0)$ & 1.93 & $0.88-4.27$ & 0.10 \\
\hline & Missing & $11(0.9)$ & $3(3.4)$ & - & & \\
\hline \multirow[t]{3}{*}{ Lymph nodes $>3 \mathrm{~cm}$} & No & $1078(92.7)$ & $74(83.1)$ & Ref. & & \\
\hline & Yes & $70(6.0)$ & 15 (16.9) & 2.32 & $1.22-4.39$ & 0.01 \\
\hline & Missing & $15(1.3)$ & - & - & & \\
\hline
\end{tabular}

${ }^{a}$ Multiple cox regression of time to TE event for baseline clinical characteristics.

Table 3

Cox regression analysis of time to TE event.

\begin{tabular}{|c|c|c|c|c|c|c|}
\hline \multirow[t]{2}{*}{ SNP } & \multicolumn{3}{|c|}{ Single-SNP } & \multicolumn{3}{|c|}{ Multiple-SNP } \\
\hline & HR & $95 \% \mathrm{CI}$ & $\mathrm{p}$-Value & HR & $95 \% \mathrm{CI}$ & p-Value \\
\hline F5 rs6025 & 1.06 & $0.44-2.55$ & 0.89 & 1.16 & $0.48-2.83$ & 0.75 \\
\hline$A B O$ rs8176719 & 0.99 & $0.75-1.37$ & 0.94 & 1.03 & $0.76-1.40$ & 0.83 \\
\hline$F G G$ rs2066865 & 1.36 & $0.98-1.89$ & 0.065 & 1.37 & $0.99-1.91$ & 0.058 \\
\hline F11 rs2036914 & 1.51 & $1.11-2.06$ & 0.009 & 1.52 & $1.11-2.07$ & 0.009 \\
\hline
\end{tabular}

Table 4

Genotype distribution of F5 rs6025 according to age.

\begin{tabular}{llll}
\hline \multirow{2}{*}{$\begin{array}{l}\text { F5 rs6025 } \\
\text { genotype }\end{array}$} & \multicolumn{2}{l}{ Proportion of patients with TE (\%) } \\
\cline { 2 - 4 } & $1.0-9.9$ years & $10.0-17.9$ years & $18.0-45.9$ years \\
\hline CC & $32 / 838(3.8)$ & $31 / 214(14.5)$ & $21 / 122(17.2)$ \\
CT & $2 / 54(3.7)$ & $\begin{array}{l}3 / 16(18.8) \\
0 / 1(0)\end{array}$ & $\begin{array}{l}0 / 5(0) \\
\text { TT }\end{array}$ \\
\hline
\end{tabular}

CI 6.7-15.5), compared with 66 of 1053 patients with $<3$ risk alleles (cumulative incidence $6.3 \%, 95 \%$ CI 4.8-7.8). Including an age group*genetic risk score interaction term to the cox model showed a significant interaction between the 2-SNP genetic risk score and the adolescent age group of 10.0-17.9 years (p 0.04).

Stratified analysis by age group, controlling for the first two genetic principal components, showed the strongest association of the 2-SNP genetic risk score in adolescents, 10.0-17.9 years (HR 1.64 per risk allele, 95\% CI 1.17-2.89, p 0.004). Twelve of 41 adolescents with $\geq 3$ risk alleles developed TE (cumulative incidence 29.3\%, 95\% CI 15.2-43.4), compared with 22 of 190 with $<3$ risk alleles (cumulative incidence $11.2 \%, \quad 95 \%$ CI 6.7-15.7) (Fig. 3b). In adults, 18.0-45.9 years, the cumulative incidence of TE was high in both the group with $\geq 3$ risk alleles $(22.1 \%, 95 \%$ CI $0-45.2)$ and with $<3$ risk alleles (16.1\%, 95\% CI 9.3-22.9) (Fig. 3c). In children, 1.0-9.9 years, the cumulative incidence of TE was low in both the group with $\geq 3$ risk alleles (4.9\%, 95\% CI 1.4-8.4) and with $<3$ risk alleles $(3.6 \%, 95 \%$ CI 2.3-4.9) (Fig. 3a). Including $F 5$ rs6025 or $A B O$ rs8176719 in the genetic risk score did not significantly improve the model. An unweighted genetic risk score using all four SNPs showed a weaker, but still significant association with TE (HR 1.28, 95\% CI 1.07-1.54, p 0.008).

\section{Discussion}

In this prospective, longitudinal cohort of patients with ALL treated according to the NOPHO ALL2008 protocol we found a significant association with TE for F11 rs2036914 and a borderline significant association with FGG rs2066865, but no significant association for F5 rs6025 or $A B O$ rs8176719. We found a significant interaction between the 2-SNP genetic risk score and age group, indicating that age is a modifier of the effect of the SNPs on the risk of TE, with adolescents having the strongest risk. These SNPs, with the exception of F5 rs6025, have not previously been tested in patients with ALL.

There is strong evidence from adult studies of SNPs from the F5, $F 11, F G G$, and $A B O$ genes being associated with TE, including a metaanalysis of GWAS from the INVENT consortium from 2015 [3], comprising 7507 venous TE cases and 52,632 controls, and a GWAS in the UK Biobank from 2017 [19], with 3290 venous TE cases and 116,868 controls. Several studies have used these SNPs as part of genetic risk scores for TE in adults [5,20,21].

The majority of the patients in our study population were children $<18$ years old. In 2017 Ruhle et al. [22] reported a GWAS of 212 nuclear families with pediatric venous TE; identifying a region on chromosome 6 as a new susceptibility locus for TE in children. They also found associations from the $A B O$ and $F 11$ loci that almost reached genome-wide significance. A study from 2009 by Nowak-Göttl et al. [23] on 244 families of children with venous TE and 268 families of children with nonvascular thromboembolic stroke found an association with haplotypes of $F G A$ and $F G G$, and with $F 5$ rs6025. In children with ALL, the few studies on inherited thrombophilia, including F5 rs6025, associated with TE are largely contradictory or inconclusive due to small numbers [24-27]. In the present study, we did not find an association for F5 rs6025 despite excellent power (96\%) to detect an association of the same magnitude as found in literature. However, the $95 \%$ CI does span clinically significant values.

The effect of non-O blood group on thrombotic vascular disease is thought to be primarily through the effect of increased plasma levels of von Willebrand factor, and consequently factor VIII [28]. A meta-analysis from 2012 found a significant association between venous TE and non-O blood group in adults [29]. There have been several reports of associations between non-O blood group and TE in children with ALL. Mizrahi et al. [30] found an association with non-O blood group in a retrospective study of 523 children with ALL of which 56 developed TE and Athale and al [24] reported an association with age and non-O blood group in a prospective cohort of 131 children with ALL of which 20 developed TE. We did not have data on blood group in our population; however, we found no association between $A B O$ rs8176719 and 

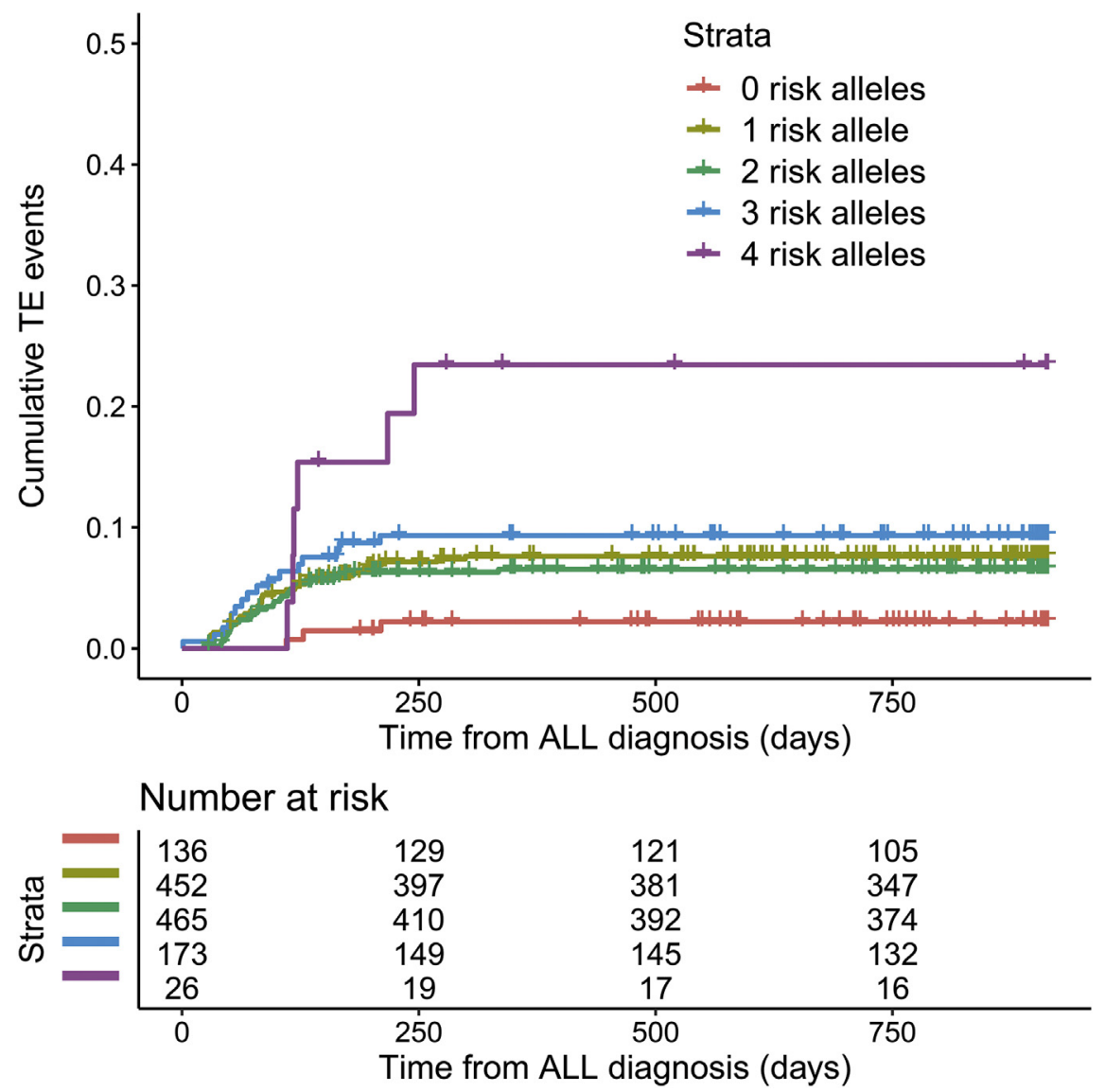

Fig. 2. Cumulative incidence of TE according to the 2-SNP genetic risk score.

Cumulative incidence of TE per number of risk alleles of the 2-SNP genetic risk score based on F11 rs2036914 and FGG rs2066865, gray's test p-value 0.003.

TE development, despite this large prospective cohort being relatively well powered (74\%) to detect an effect of the same magnitude as found in the literature of TE in adults.

Factor XI is a component of the coagulation pathway and elevated levels have been associated with increased TE development in adults [31], while fibrinogen is the precursor to fibrin, which is required to stabilize the coagulation clot in hemostasis, and fibrinogen gamma makes up part of the fibrinogen molecule. Different levels of isoforms of the fibrinogen gamma chain are likely to be associated with TE development, although this has recently been questioned [10,32]. In exploratory analysis we created a 2-SNP genetic risk score based on F11 rs2036914 and FGG rs2066865 and demonstrated an interaction between the genetic risk score and age group. Several previous studies have shown that adolescents are at increased risk of TE compared with younger children [33]. In children and adolescents, the hemostatic system is under constant development and the levels of many
A
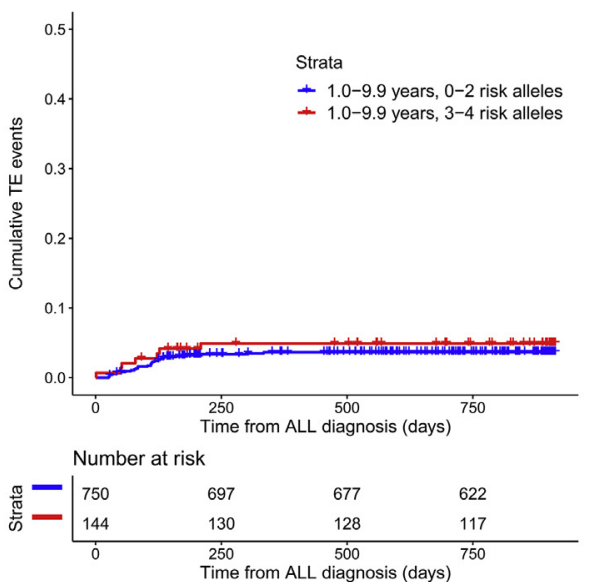

B
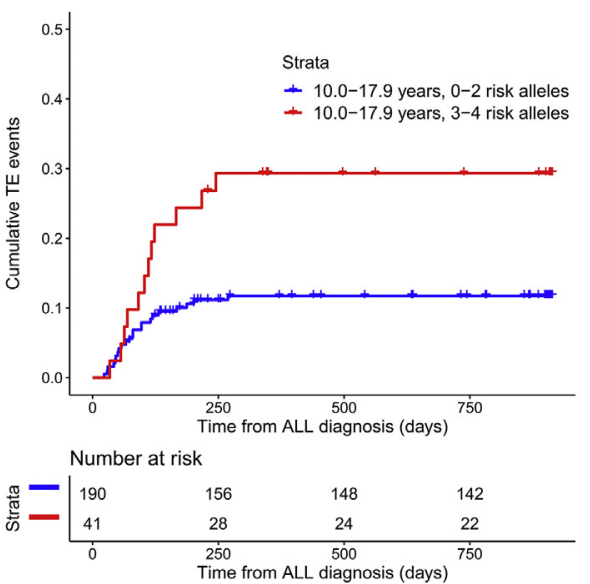

C
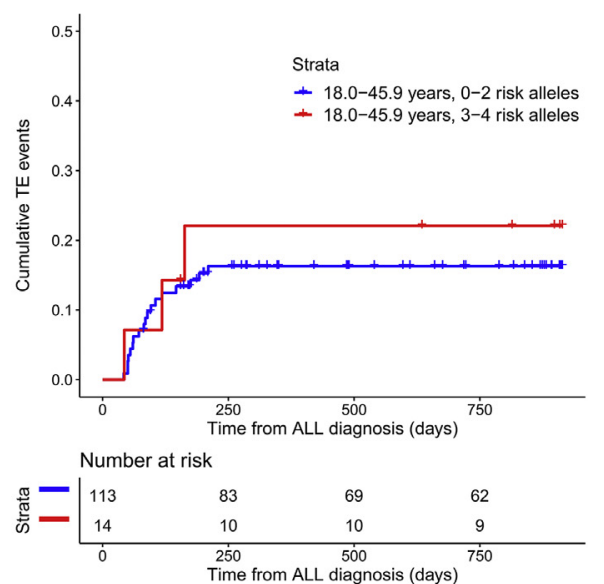

Fig. 3. Cumulative incidence of TE by number of risk alleles and age group.

Incidence of TE in patients with $\geq 3$ (red) or $<3$ (blue) risk alleles of the 2-SNP genetic risk score ( $F 11$ rs2036914 and FGG rs2066865) for children 1.0-9.9 years (A), adolescents 10.0-17.9 years (B), and adults 18.0-45.9 years old (C) respectively. (For interpretation of the references to colour in this figure legend, the reader is referred to the web version of this article.) 
components of the hemostatic system are different to those of adults [34,35]. For instance, studies have found lower levels of factor XI in healthy adolescents compared with adults $[35,36]$. In addition, the hormonal changes occurring during puberty may also affect the risk of TE, though there is little data on this subject. Greiner et al. [37] recently showed that prophylactic antithrombotic treatment significantly reduced the incidence of TE in patients with ALL. If validated, a F11 rs2036914 and FGG rs2066865 risk prediction model should be tested as a stratification tool for prophylactic antithrombotic interventions in patients with ALL.

The strengths of this study include the large population-based cohort of patients with ALL from 1 to 45 years of age, treated according to a uniform and strictly regulated research-protocol. The NOPHO ALL2008 protocol has a high inclusion rate and the prospective toxicity registration has a reporting compliance of $98.9 \%$ [2]. In addition, we performed a priori power calculations before performing our SNP analyses. A potential weakness of the study is the delay of consent to participation in the genetic studies following the ALL diagnosis in some cases; thus there may have been cases of early deaths that were not included and therefore could not be accounted for as competing events.

Second, only $53.9 \%$ of the TE events were non-CVL-related. It would be interesting to investigate whether the selected SNPs play a more important role when excluding the mechanic effect of the CVL. However, we did not have large enough numbers for this.

Third, despite having a large multinational cohort, we did not have enough power to test more individual SNPs; particularly those with smaller effect sizes or lower minor allele frequencies. Two of our SNPs were not available in the Illumina chip and were imputed. Imputation of SNPs with low risk allele frequencies is difficult, which is clearly shown in the poor quality score for $F 2$ rs1799963, forcing us to exclude this SNP.

In conclusion, we find a significant association with TE for F11 rs2036914 and a trend toward significant association for $F G G$ rs2066865 in patients with ALL. The role of F11 rs2036914 and FGG rs2066865 for TE risk prediction models in ALL patients should be validated, particularly in adolescents, before applying these in preventive strategies to avoid TE.

\section{Declaration of competing interest}

None.

\section{Acknowledgements}

We would like to thank all the patients included in the study and our colleagues at the ALL treatment centers for reporting data into the NOPHO ALL registry and completing the TE registration forms. The SNP analyses of the NOPHO patients was supported by The Danish Cancer Society, The Danish Childhood Cancer Foundation, The Swedish Childhood Cancer Foundation, The Nordic Cancer Union, The Otto Christensen Foundation, University Hospital Rigshospitalet, and The Novo Nordic Foundation. The study was supported by research grant from the South-Eastern Norway Regional Health Authority, Norway [grant number 2016011].

\section{Appendix A. Supplementary data}

Supplementary data to this article can be found online at https:// doi.org/10.1016/j.thromres.2019.11.002.

\section{References}

[1] C.U. Rank, N. Toft, R. Tuckuviene, K. Grell, O.J. Nielsen, T.L. Frandsen, H.V.H. Marquart, B.K. Albertsen, U. Tedgard, H. Hallbook, E. Ruud, K.B. Jarvis, P. Quist-Paulsen, P. Huttunen, U. Wartiovaara-Kautto, O.G. Jonsson, S.S. Trakymiene, L. Griskevicius, K. Saks, M. Punab, K. Schmiegelow,
Thromboembolism in acute lymphoblastic leukemia: results of NOPHO ALL2008 protocol treatment in patients 1-45 years, Blood 131 (22) (2018) 2475-2484.

[2] T.L. Frandsen, M. Heyman, J. Abrahamsson, K. Vettenranta, A. Asberg, G. Vaitkeviciene, K. Pruunsild, N. Toft, H. Birgens, H. Hallbook, P. Quist-Paulsen, L. Griskevicius, L. Helt, B.V. Hansen, K. Schmiegelow, Complying with the European Clinical Trials directive while surviving the administrative pressure - an alternative approach to toxicity registration in a cancer trial, Eur. J. Cancer 50 (2) (2014) 251-259.

[3] M. Germain, D.I. Chasman, H. de Haan, W. Tang, S. Lindstrom, L.C. Weng, M. de Andrade, M.C. de Visser, K.L. Wiggins, P. Suchon, N. Saut, D.M. Smadja, G. Le Gal, A. van Hylckama Vlieg, A. Di Narzo, K. Hao, C.P. Nelson, A. Rocanin-Arjo, L. Folkersen, R. Monajemi, L.M. Rose, J.A. Brody, E. Slagboom, D. Aissi, F. Gagnon, J.F. Deleuze, P. Deloukas, C. Tzourio, J.F. Dartigues, C. Berr, K.D. Taylor, M. Civelek, P. Eriksson, B.M. Psaty, J. Houwing-Duitermaat, A.H. Goodall, F. Cambien, P. Kraft, P. Amouyel, N.J. Samani, S. Basu, P.M. Ridker, F.R. Rosendaal, C. Kabrhel, A.R. Folsom, J. Heit, P.H. Reitsma, D.A. Tregouet, N.L. Smith, P.E. Morange, Meta-analysis of 65,734 individuals identifies TSPAN15 and SLC44A2 as two susceptibility loci for venous thromboembolism, Am. J. Hum. Genet. 96 (4) (2015) 532-542.

[4] H.G. de Haan, I.D. Bezemer, C.J. Doggen, S. Le Cessie, P.H. Reitsma, A.R. Arellano, C.H. Tong, J.J. Devlin, L.A. Bare, F.R. Rosendaal, C.Y. Vossen, Multiple SNP testing improves risk prediction of first venous thrombosis, Blood 120 (3) (2012) 656-663.

[5] A.R. Folsom, W. Tang, L.C. Weng, N.S. Roetker, M. Cushman, S. Basu, J.S. Pankow, Replication of a genetic risk score for venous thromboembolism in whites but not in African Americans, J. Thromb. Haemost. 14 (1) (2016) 83-88.

[6] V.S. Blanchette, L.R. Brandao, V.R. Breakey, S. Revel-Vilk, SickKids Handbook of Pediatric Thrombosis and Hemostasis, Karger, Basel, Switzerland, 2017.

[7] F. Yamamoto, H. Clausen, T. White, J. Marken, S. Hakomori, Molecular genetic basis of the histo-blood group ABO system, Nature 345 (6272) (1990) 229-233.

[8] J.A. Heit, S.M. Armasu, Y.W. Asmann, J.M. Cunningham, M.E. Matsumoto, T.M. Petterson, M. De Andrade, A genome-wide association study of venous thromboembolism identifies risk variants in chromosomes 1q24.2 and 9q, J. Thromb. Haemost. 10 (8) (2012) 1521-1531.

[9] M.M. Gorski, H.G. de Haan, I. Mancini, L.A. Lotta, P. Bucciarelli, S.M. Passamonti, A. Cairo, E. Pappalardo, A. van Hylckama Vlieg, I. Martinelli, F.R. Rosendaal, F. Peyvandi, Next-generation DNA sequencing to identify novel genetic risk factors for cerebral vein thrombosis, Thromb. Res. 169 (2018) 76-81.

[10] S. Uitte de Willige, M.C. de Visser, J.J. Houwing-Duistermaat, F.R. Rosendaal, H.L. Vos, R.M. Bertina, Genetic variation in the fibrinogen gamma gene increases the risk for deep venous thrombosis by reducing plasma fibrinogen gamma' levels, Blood 106 (13) (2005) 4176-4183.

[11] Y. Li, I.D. Bezemer, C.M. Rowland, C.H. Tong, A.R. Arellano, J.J. Catanese, J.J. Devlin, P.H. Reitsma, L.A. Bare, F.R. Rosendaal, Genetic variants associated with deep vein thrombosis: the F11 locus, J. Thromb. Haemost. 7 (11) (2009) 1802-1808.

[12] N. Toft, H. Birgens, J. Abrahamsson, P. Bernell, L. Griskevicius, H. Hallbook, M. Heyman, M.S. Holm, E. Hulegardh, T.W. Klausen, H.V. Marquart, O.G. Jonsson, O.J. Nielsen, P. Quist-Paulsen, M. Taskinen, G. Vaitkeviciene, K. Vettenranta, A. Asberg, K. Schmiegelow, Risk group assignment differs for children and adults 1-45 yr with acute lymphoblastic leukemia treated by the NOPHO ALL-2008 protocol, Eur. J. Haematol. 90 (5) (2013) 404-412.

[13] S. Ranta, R. Tuckuviene, A. Makipernaa, B.K. Albertsen, T. Frisk, U. Tedgard, O.G. Jonsson, K. Pruunsild, N. Gretenkort Andersson, M. Winther Gunnes, S. Saulyte Trakymiene, T. Frandsen, M. Heyman, E. Ruud, J. Helgestad, Cerebral sinus venous thromboses in children with acute lymphoblastic leukaemia - a multicentre study from the Nordic Society of Paediatric Haematology and Oncology, Br. J. Haematol. 168 (4) (2015) 547-552.

[14] N. Toft, H. Birgens, J. Abrahamsson, L. Griskevicius, H. Hallbook, M. Heyman, T.W. Klausen, O.G. Jonsson, K. Palk, K. Pruunsild, P. Quist-Paulsen, G. Vaitkeviciene, K. Vettenranta, A. Asberg, L.R. Helt, T. Frandsen, K. Schmiegelow, Toxicity profile and treatment delays in NOPHO ALL2008-comparing adults and children with Philadelphia chromosome-negative acute lymphoblastic leukemia, Eur. J. Haematol. 96 (2) (2016) 160-169.

[15] C.A. Anderson, F.H. Pettersson, G.M. Clarke, L.R. Cardon, A.P. Morris, K.T. Zondervan, Data quality control in genetic case-control association studies, Nat. Protoc. 5 (9) (2010) 1564-1573.

[16] K. Owzar, Z. Li, N. Cox, S.H. Jung, Power and sample size calculations for SNP association studies with censored time-to-event outcomes, Genet. Epidemiol. 36 (6) (2012) 538-548.

[17] P.E. Morange, P. Suchon, D.A. Tregouet, Genetics of venous thrombosis: update in 2015, Thromb. Haemost. 114 (5) (2015) 910-919.

[18] S.E. Hunt, W. McLaren, L. Gil, A. Thormann, H. Schuilenburg, D. Sheppard, A. Parton, I.M. Armean, S.J. Trevanion, P. Flicek, F. Cunningham, Ensembl variation resources, Database (Oxford) 2018, 2018.

[19] D. Klarin, C.A. Emdin, P. Natarajan, M.F. Conrad, S. Kathiresan, Genetic analysis of venous thromboembolism in UK biobank identifies the ZFPM2 locus and implicates obesity as a causal risk factor, Circ. Cardiovasc. Genet. 10 (2) (2017).

[20] J.M. Soria, P.E. Morange, J. Vila, J.C. Souto, M. Moyano, D.A. Tregouet, J. Mateo, N. Saut, E. Salas, R. Elosua, Multilocus genetic risk scores for venous thromboembolism risk assessment, J. Am. Heart Assoc. 3 (5) (2014) e001060.

[21] M. Bruzelius, M. Bottai, M. Sabater-Lleal, R.J. Strawbridge, A. Bergendal, A. Silveira, A. Sundstrom, H. Kieler, A. Hamsten, J. Odeberg, Predicting venous thrombosis in women using a combination of genetic markers and clinical risk factors, J. Thromb. Haemost. 13 (2) (2015) 219-227.

[22] F. Ruhle, A. Witten, A. Barysenka, A. Huge, A. Arning, C. Heller, A. Krumpel, R. Mesters, A. Franke, W. Lieb, M. Riemenschneider, M. Hiersche, V. Limperger, 
U. Nowak-Gottl, M. Stoll, Rare genetic variants in SMAP1, B3GAT2, and RIMS1 contribute to pediatric venous thromboembolism, Blood 129 (6) (2017) 783-790.

[23] U. Nowak-Gottl, H. Weiler, I. Hernandez, S. Thedieck, T. Seehafer, T. Schulte, M. Stoll, Fibrinogen alpha and gamma genes and factor VLeiden in children with thromboembolism: results from 2 family-based association studies, Blood 114 (9) (2009) 1947-1953.

[24] U.H. Athale, T. Mizrahi, C. Laverdiere, T. Nayiager, Y.L. Delva, G. Foster, L. Thabane, M. David, J.M. Leclerc, A.K.C. Chan, Impact of baseline clinical and laboratory features on the risk of thrombosis in children with acute lymphoblastic leukemia: a prospective evaluation, Pediatr. Blood Cancer 65 (10) (2018) e27256.

[25] Y.A. Torun, T. Patiroglu, M.A. Ozdemir, Y. Ozkul, A. Ekici, M. Karakukcu, Inherited prothrombotic risk factors in Turkish children with acute lymphoblastic leukemia: significance of concomitant genetic mutation, Clin. Appl. Thromb. Hemost. 18 (2) (2012) 218-221.

[26] U. Nowak-Gottl, C. Wermes, R. Junker, H.G. Koch, R. Schobess, G. Fleischhack, D. Schwabe, S. Ehrenforth, Prospective evaluation of the thrombotic risk in children with acute lymphoblastic leukemia carrying the MTHFR TT 677 genotype, the prothrombin G20210A variant, and further prothrombotic risk factors, Blood 93(5) (1999) 1595-9.

[27] D.F. Akin, K. Sipahi, T. Kayaalp, Y. Egin, S. Tasdelen, E. Kurekci, U. Ezer, N. Akar, Factor V Leiden and prothrombin 20210A mutations among Turkish pediatric leukemia patients, Leuk. Res. Treat. 2012 (2012) 250432.

[28] M. Franchini, P.M. Mannucci, ABO blood group and thrombotic vascular disease, Thromb. Haemost. 112 (6) (2014) 1103-1109.

[29] F. Dentali, A.P. Sironi, W. Ageno, S. Turato, C. Bonfanti, F. Frattini, S. Crestani, M. Franchini, Non-O blood type is the commonest genetic risk factor for VTE: results from a meta-analysis of the literature, Semin. Thromb. Hemost. 38 (5) (2012) 535-548.

[30] T. Mizrahi, J.M. Leclerc, M. David, T. Ducruet, N. Robitaille, ABO group as a thrombotic risk factor in children with acute lymphoblastic leukemia: a retrospective study of 523 patients, J. Pediatr. Hematol. Oncol. 37 (5) (2015) e328-e332.

[31] A.R. Folsom, W. Tang, N.S. Roetker, S.R. Heckbert, M. Cushman, J.S. Pankow, Prospective study of circulating factor XI and incident venous thromboembolism: the longitudinal investigation of thromboembolism etiology (LITE), Am. J. Hematol. 90 (11) (2015) 1047-1051.

[32] A.R. Folsom, W. Tang, K.M. George, S.R. Heckbert, R.F. MacLehose, M. Cushman, J.S. Pankow, Prospective study of gamma' fibrinogen and incident venous thromboembolism: the longitudinal investigation of thromboembolism etiology (LITE), Thromb. Res. 139 (2016) 44-49.

[33] M. Andrew, M. David, M. Adams, K. Ali, R. Anderson, D. Barnard, M. Bernstein, L. Brisson, B. Cairney, D. DeSai, et al., Venous thromboembolic complications (VTE) in children: first analyses of the Canadian Registry of VTE, Blood 83 (5) (1994) 1251-1257.

[34] M. Andrew, P. Vegh, M. Johnston, J. Bowker, F. Ofosu, L. Mitchell, Maturation of the hemostatic system during childhood, Blood 80 (8) (1992) 1998-2005.

[35] C. Attard, T. van der Straaten, V. Karlaftis, P. Monagle, V. Ignjatovic, Developmental hemostasis: age-specific differences in the levels of hemostatic proteins, J. Thromb. Haemost. 11 (10) (2013) 1850-1854.

[36] S. Kuhle, C. Male, L. Mitchell, Developmental hemostasis: pro- and anticoagulant systems during childhood, Semin. Thromb. Hemost. 29 (4) (2003) 329-338.

[37] J. Greiner, M. Schrappe, A. Claviez, M. Zimmermann, C. Niemeyer, R. Kolb, W. Eberl, F. Berthold, E. Bergstrasser, A. Gnekow, E. Lassay, P. Vorwerk, M. Lauten, A. Sauerbrey, J. Rischewski, A. Beilken, G. Henze, W. Korte, A. Moricke, THROMBOTECT - a randomized study comparing low molecular weight heparin, antithrombin and unfractionated heparin for thromboprophylaxis during inudction therapy of acute lymphoblastic leukemia in children and adolescents, Haematologica 104 (4) (2018) 756-765. 